POS $\quad$ PROCEEDINGS

\title{
The ESS neutrino facility for CP violation discovery
}

\section{Marcos Dracos ${ }^{* \dagger}$}

IPHC, Université de Strasbourg, CNRS/IN2P3, F-67037 Strasbourg, France

E-mail: marcos.dracosein2p3. fr

The relatively large value of the neutrino mixing angle $\theta_{13}$ measured in 2012 by neutrino reactor experiments opened the door to observe for the first time a possible $\mathrm{CP}$ violation in the leptonic sector. The measured value of $\theta_{13}$ also privileges the 2 nd oscillation maximum for the discovery of $\mathrm{CP}$ violation instead of the usually used 1st oscillation maximum. The sensitivity at this 2 nd oscillation maximum is about three times higher than for the 1 st oscillation maximum inducing a lower sensitivity to systematic errors. Going to the 2 nd oscillation maximum requires a very intense neutrino beam with the appropriate energy. The world's most intense pulsed spallation neutron source, the European Spallation Source, has a proton linac with $5 \mathrm{MW}$ power and $2 \mathrm{GeV}$ energy. This linac also has the possibility to become the proton driver of the world's most intense neutrino beam with very high potentiality to discover a neutrino CP violation. The physics performance of this neutrino Super Beam in conjunction with a megaton Water Cherenkov neutrino detector installed $1000 \mathrm{~m}$ down in a mine at a distance of about $500 \mathrm{~km}$ from ESS has been evaluated. In addition, the choice of such voluminous detector will extent the physics program to proton-decay, atmospheric neutrinos and astrophysics searches. The ESS proton linac upgrades, the target station optimization and the physics potential are described. In addition to neutrinos, this facility will also produce at the same time a copious number of muons which could be used by a low energy nuSTORM facility, a future neutrino factory or a muon collider. The ESS neutron installations, under construction, will be fully operational by 2023 at which moment the upgrades for the neutrino facility could start. This project is now supported by the COST Action CA15139 "Combining forces for a novel European facility for neutrino-antineutrino symmetry-violation discovery" (EuroNuNet).

38th International Conference on High Energy Physics

3-10 August 2016

Chicago, USA

\footnotetext{
* Speaker.

${ }^{\dagger}$ On behalf of the ESS $v$ SB project.
} 


\section{Introduction}

The already matter-antimatter asymmetry measured in the hadronic sector is by several orders of magnitude insufficient to contribute to the explanation of the antimatter disappearance in the Univers [1]. To understand this asymmetry other CP Violation sources are investigated. In 2012 reactor experiments $[2,3,4]$ have measured a relatively high $\theta_{13}$ mixing angle in neutrino oscillations. This measurement opens now the way to a possible CP Violation observation in the leptonic sector, which could explain (under some conditions) the matter-antimatter asymmetry in the Universe.

For CP Violation in the neutrino sector new next generation accelerator neutrino projects are proposed. These projects are based on very intense neutrino beams using MW class proton drivers. The performance of these projects strongly depends on the systematic uncertainties which could be achieved by the moment of the operation of the relating experiments. One of these projects, ESSvSB [5], proposes to use the very powerful proton linac, under construction, of the European Spallation Source (ESS) [6] to produce a high intensity muon neutrino beam, which coulb be sent towards a megaton Water Cherenkov detector. This detector could be placed at a distance corresponding to the second oscillation maximum in order to observe a neutrino CP Violation. Placing the far detector at the second oscillation maximum compared to the first one, procures enhanced sensitivity to observe a possible CP Violation, with less sensitivity to systematic errors [7].

The ESS $v$ SB facility would also produce a copious number of muons which could be used by other projects as for a muon collider.

\section{ESS as Neutrino Facility}

The ESS neutron facility is under construction in Lund, Sweden, since 2014. It will produce neutrons for many applications going from solid physics up to biology. The proton linac used to produce the spallation neutrons of the facility has a duty cycle of only $4 \%$ (pulse duration of $2.86 \mathrm{~ms}$ ). By doubling the pulse frequency from $14 \mathrm{~Hz}$ to $28 \mathrm{~Hz}$ (other operation scenarios are also possible) and thus doubling the mean proton power from $5 \mathrm{MW}$ to $10 \mathrm{MW}$, this facility could be used at the same time to produce neutrons and neutrinos. The main characteristics of this linac are given in Table 1. The number of protons on target per year (208 days) is of the order of $2.7 \times 10^{23}$. The proton kinetic energy is $2.0 \mathrm{GeV}$ and it could be go up to $3.6 \mathrm{GeV}$ in future upgrades using empty space left in purpose in the linac tunnel.

The proton beam extracted from the linac can be sent to a target followed by a hadronic collector to produce muon neutrinos from pion decays. For the hadronic collector, focusing the produced pions towards the far neutrino detector, a classical magnetic horn can be used. In order to produce the necessary magnetic field inside the horn, a very high pulsed current ( $2350 \mathrm{kA})$ has to be sent. These pulses have to be as short as few $\mu$ s for power dissipation reasons. For mainly these reason, the proton pulse duration of $2.86 \mathrm{~ms}$ has to be considerably reduced. To perform this, a proton accumulation ring of a circumference of about $400 \mathrm{~m}$ is needed. This brings another extra requirement, which is the utilization of $\mathrm{H}^{-}$in the linac instead of protons. Indeed, in order to be able to inject protons inside the ring while already other protons circulate in, $\mathrm{H}^{-}$are needed to be injected coming from the proton linac and striped at the entrance of the ring. This brings additional cost to 
Table 1: Main ESS facility parameters of the proton beam.

\begin{tabular}{lc}
\hline Parameter & Value \\
\hline Average beam power & $5 \mathrm{MW}$ \\
Proton kinetic energy & $2.0 \mathrm{GeV}$ \\
Average macro-pulse current & $62.5 \mathrm{~mA}$ \\
Macro-pulse length & $2.86 \mathrm{~ms}$ \\
Pulse repetition rate & $14 \mathrm{~Hz}$ \\
Maximum accelerating cavity & \\
surface field & $45 \mathrm{MV} / \mathrm{m}$ \\
Maximum linac length (excluding & \\
contingency and upgrade space) & $352.5 \mathrm{~m}$ \\
Annual operating period & $5000 \mathrm{~h}$ \\
Reliability & $95 \%$ \\
\hline
\end{tabular}

this facility which could be avoided in case other hadronic collectors are found not needing high current pulses (e.g. based on superconducting solenoids).

A target station can be placed just after the accumulation ring followed by a short decay tunnel ( $\sim 25 \mathrm{~m}$ length). In oder to mitigate the high power proton beam shock on the target which could produce violent vibrations and power dissipation problems, four identical target/horn systems could be used pulsed alternatively, solution already proposed by the FP7 Design Study EUROv [8, 9]. For this, a beam switchyard can be used at the exit of the accumulator to distribute the beam to the four systems. At the end of the decay tunnel a beam dump has to be installed to stop all remaining particles, mainly protons, pions and muons. A near detector, to monitor the unoscillated neutrino beam and to measure the relevant neutrino cross-sections, can be installed at about $500 \mathrm{~m}$ from the neutrino target.

Fig. 1 presents a schematic view of the ESS neutron and neutrino facility while Fig. 2 presents the neutrino spectrum which can be obtained at an arbitrary distance of $100 \mathrm{~km}$ for one year running. A small contamination of the muon neutrino beam from $0.5 \%$ electron neutrinos, polluting the signal at the level of the far detector, could be used by a "smart" near detector to measure the electron neutrino cross-section at the neutrino energies used by the project.

\section{The neutrino oscillation project}

For this project a location for the voluminous far detector has to be found. In order to keep the excavation cost of the underground laboratory to host such a detector at a reasonable level, it would be better to located it in an existing mine. At a distance of $540 \mathrm{~km}$ the active mine of Garpenberg (north of Lund, Sweden) could host this megaton-class Water Cherenkov detector [10]. Taking into account the neutrino spectrum and the distance between Lund and Garpenberg, the detector would be, as requested, on the second oscillation maximum. Fig. 3 presents the $v_{\mu} \rightarrow v_{e}$ oscillation probability as a function of the neutrino energy together with neutrino energy distribution of detected by the far detector electron neutrinos coming from muon neutrino oscillations. This shows 


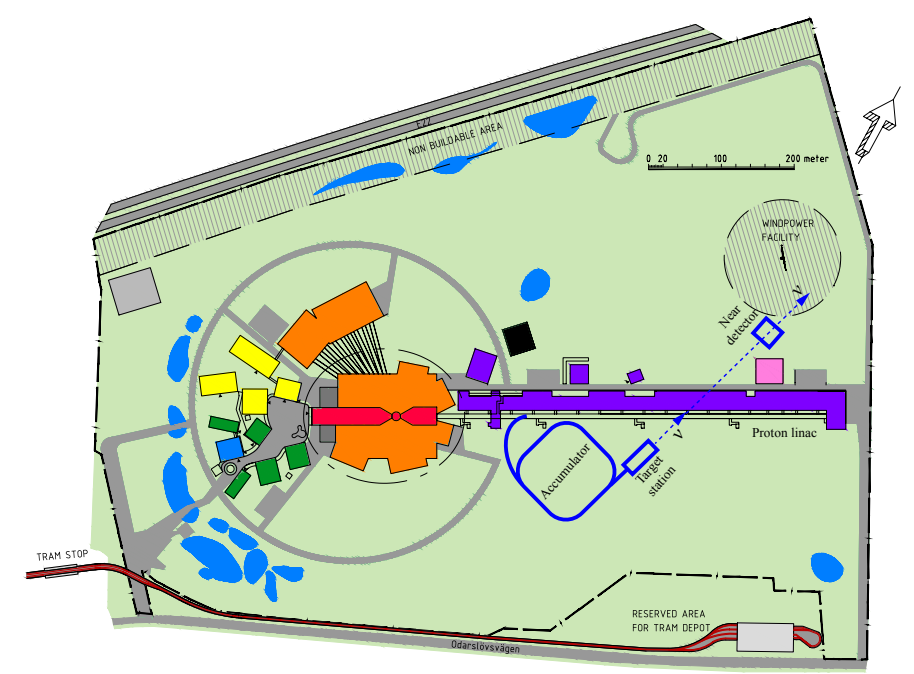

Figure 1: Schematic view of the ESS neutron and neutrino facility. The neutrino facility at the ESS site is composed, on top of the proton linac, of an accumulator, a target station, a decay tunnel and a near neutrino detector.
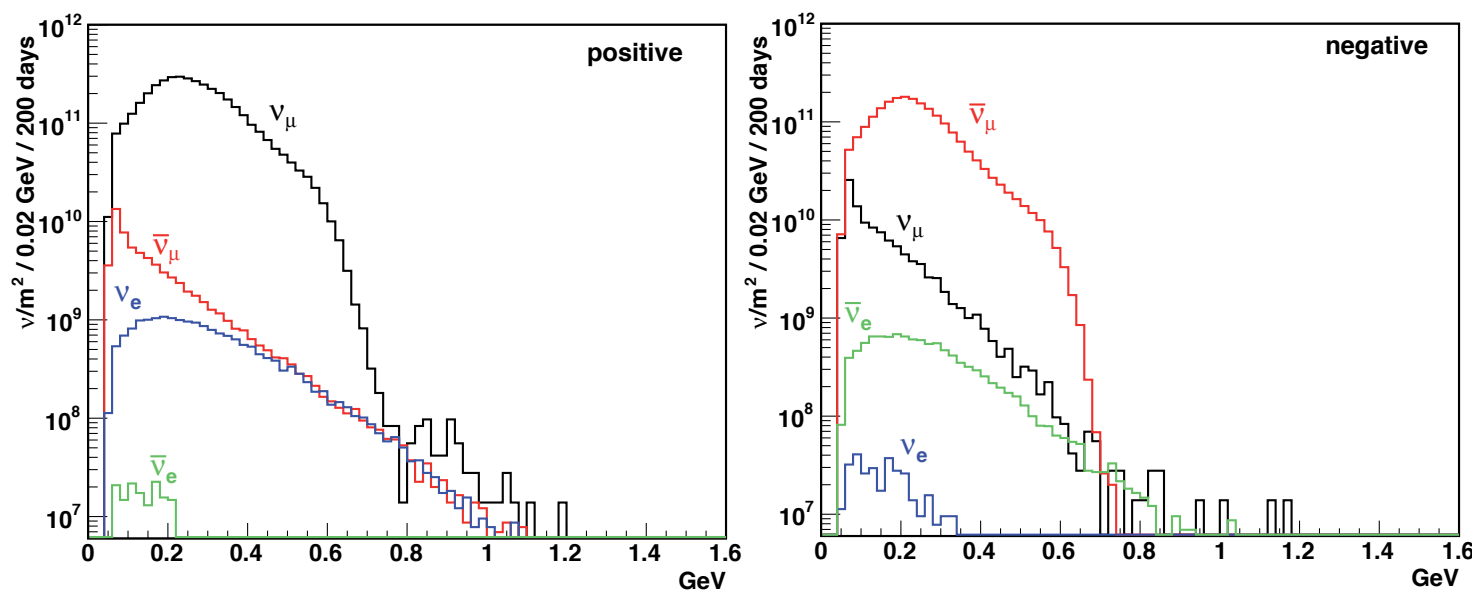

Figure 2: Neutrino energy spectrum for "neutrinos" (left, positive current in the horn) and "antineutrinos" (right, positive current in the horn) runs at a distance of $100 \mathrm{~km}$ on-axis from the target station, for $2.0 \mathrm{GeV}$ protons.

that the ESS $v$ SB facility operates almost exclusively on the second oscillation maximum. In 10 years data taking, ESSvSB would collect about 300 electron neutrinos (2 years running) and 250 electron antineutrinos ( 8 years running) coming from muon neutrino oscillations.

Fig. 4 presents the fraction of the full parameter $\delta_{C P}$ range as a function of the baseline for $3 \sigma$ and $5 \sigma \mathrm{CP}$ violation discovery significance. If the proton energy remains $2 \mathrm{GeV}$ (present design), the best baseline is around $400 \mathrm{~km}$, close to the Zinkgrouvan mine (360 km). The Garpenberg mine $(540 \mathrm{~km})$ has a better potentiality in case the proton energy is increased above $2.5 \mathrm{GeV}$ and can cover up to $60 \%$ of the $\delta_{C P}$ range for a $5 \sigma$ significance. This performance is obtained assuming an unknown mass hierarchy and 5\% systematic error on signal and $10 \%$ on background. It has to be noted that the first oscillation maximum is around $180 \mathrm{~km}$ with significantly less $\delta_{C P}$ coverage. 
The obtained $\delta_{C P}$ coverage is limited by the statistics and not by systematics. Increasing the data taking period would increase the $\delta_{C P}$ coverage. For lower systematic uncertainties, going to the first oscillation maximum could probably be more interesting but also more risky because of the high uncertainty on achieving by that time the necessary systematic errors.

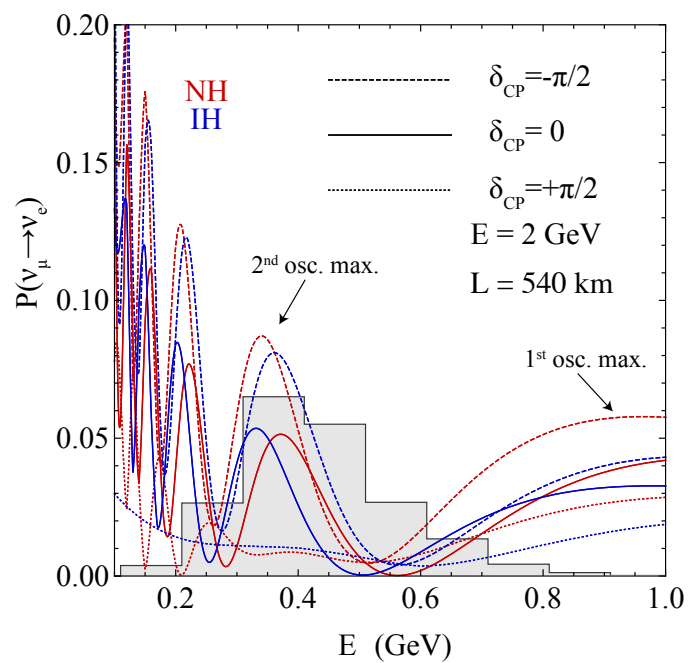

Figure 3: $v_{\mu} \rightarrow v_{e}$ oscillation probability as a function of the neutrino momentum. The shaded distribution is the momentum distribution of electron neutrinos as they would be detected by the far detector.

\section{Muon Facility}

At the level of the beam dump of the neutrino facility a huge number of muons can be collected for other applications. These pions and muons are supposed to be stopped by the neutrino tunnel beam dump. The purpose of stopping these muons is not to leave them decay and contaminate the muon neutrino beam with more electron neutrinos. Fig. 5 presents the momentum distribution of the non-decayed pions and muons at the level of the beam dump. The mean momentum of the pions is $0.71 \mathrm{GeV}$ while the one of muons is of the order of $0.46 \mathrm{MeV}$ while. An adequate collecting device could be placed at the place of the beam dump to deviate and collect all these muons and part of the non-decayed pions which could give more muons during the extraction. More than $4 \times 10^{20}$ muons per year can be extracted. These muons can be used for a "low" nuSTORM neutrino experiments [11] and for R\&D for 6D muon cooling and studies for a possible future muon collider. It has been shown that a Higgs Factory based on an ESS muon collider could produce more than half a million Higgs bosons per year [12], enough to study in details this particle.

\section{Aknowledgements}

This project is supported by the COST Action CA15139 "Combining forces for a novel European facility for neutrino-antineutrino symmetry-violation discovery" (EuroNuNet) as well as by EU Regional Structural Funds in the region in Sweden where the neutrino detector could be located. 

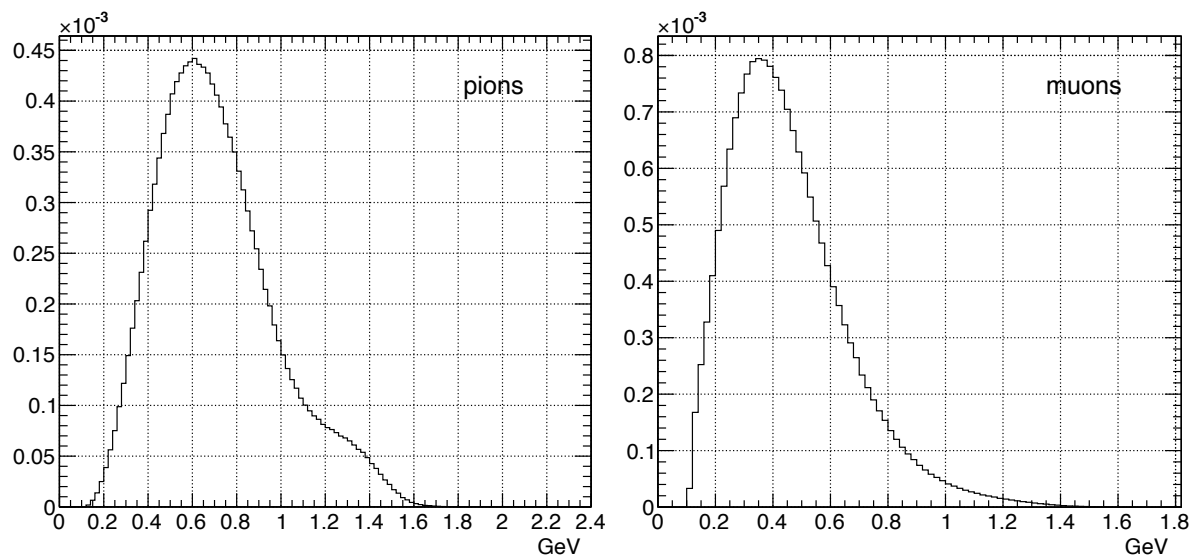

Figure 5: Pion and muon momentum distribution per proton at the level of the neutrino facility beam dump.

\section{References}

[1] C. Patrignani et al. [Particle Data Group Collaboration], Chin. Phys. C 40 (2016) no.10, 100001. doi:10.1088/1674-1137/40/10/100001.

[2] F. P. An et al. [DAYA-BAY Collaboration], "Observation of electron-antineutrino disappearance at Daya Bay,” Phys. Rev. Lett. 108, 171803 (2012) [arXiv:1203.1669 [hep-ex]].

[3] J. K. Ahn et al. [RENO Collaboration], "Observation of Reactor Electron Antineutrino Disappearance in the RENO Experiment,” Phys. Rev. Lett. 108, 191802 (2012) [arXiv:1204.0626 [hep-ex]].

[4] Y. Abe et al. [DOUBLE-CHOOZ Collaboration], "Indication for the disappearance of reactor electron antineutrinos in the Double Chooz experiment," Phys. Rev. Lett. 108, 131801 (2012) [arXiv:1112.6353 [hep-ex]].

[5] "A Very Intense Neutrino Super Beam Experiment for Leptonic CP Violation Discovery based on the European Spallation Source Linac”, Nuclear Physics B, Volume 885, August 2014, 127-149.

[6] The European Spallation Source, http://europeanspallationsource.se/, ESS TDR, Release 1.0, Nov. 2012.

[7] "Optimization of neutrino oscillation facilities for large $\theta_{13}$," JHEP 1204, 089 (2012).

[8] T. R. Edgecock, O. Caretta, T. Davenne, C. Densham, M. Fitton, D. Kelliher, P. Loveridge and S. Machida et al., "The EUROnu Project," Phys. Rev. ST Accel. Beams 16, 021002 (2013) [arXiv:1305.4067 [physics.acc-ph]].

[9] E. Baussan et al. [EUROnu Super Beam Collaboration], "The SPL-based Neutrino Super Beam," Phys. Rev. ST Accel. Beams 17, 031001 (2014), arXiv:1212.0732 [physics.acc-ph].

[10] "Study of the performance of a large scale water-Cherenkov detector (MEMPHYS)," JCAP 1301 (2013) 024.

[11] "nuSTORM - Neutrinos from STORed Muons: Letter of Intent to the Fermilab Physics Advisory Committee," arXiv:1206.0294 [hep-ex].

[12] Seminar at Uppsala University, C. Rubbia, February 2016. 